\title{
Growth properties of Eden model with acceleration sites
}

\author{
Sasuke Miyazima ${ }^{\mathrm{a}, \mathrm{b}, *}$, Hiroyuki Yoshinaga ${ }^{\mathrm{a}}$, Tomomasa Nagamine ${ }^{\mathrm{a}}$, \\ Hiroshi Takano ${ }^{c}$, Janos Kertész ${ }^{d}, H$. Eugene Stanley ${ }^{b}$ \\ a Department of Engineering Physics, Chubu University, Kasugai, Aichi 487, Japan \\ ${ }^{\mathrm{b}}$ Center for Polymer Studies, Boston University, Boston, MA 02215, USA \\ ${ }^{\mathrm{c}}$ Kochi National College of Technology, Nankoku, Kochi 783, Japan \\ ${ }^{\mathrm{d}}$ Institute of Physics, Technical University of Budapest, Budapest, Budafoki ut 8, H-1111, Hungary
}

Received 2 January 1998

\begin{abstract}
One plus one dimensional growth of an Eden model with acceleration sites is investigated by simulations, where the acceleration sites which are distributed at random before the process starts become immediately Eden cells if the surface of Eden cluster touches them. The critical concentration of acceleration sites where the growth rate of the average cluster height diverges is found as $p_{c}=0.592 \pm 0.005$ corresponding to the site percolation threshold of the square lattice. The exponent which characterizes this divergence near the percolation threshold have been found as $v=1.33 \pm 0.08$. An effective roughness exponent $\alpha$ which characterizes the surface morphology is found to belong to the same universality class as the Eden model for $p<p_{c}$. At the critical concentration, the present system changes to hold a self-similar surface. (C) 1998 Elsevier Science B.V. All rights reserved.
\end{abstract}

\section{Introduction}

Rough surfaces of growth models [1,2] such as ballistic deposition model [3], Eden model [4-7] are important subjects in fractal studies. The $1+1$ dimensional growth of Eden model is a prototype to study a rough surface (here $1+1$ refers to one-dimensional substrate and one growth dimension, i.e. the resulting cluster is two dimensional).

Originally, the Eden model was introduced in order to study a two-dimensional formation of tumor starting from a single seed [4]. Family and Vicsek discussed the dynamics of scaling theory for one-dimensional growth of Eden model starting from

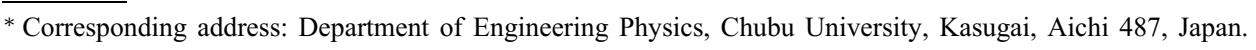


seeds on a line [8]. The fluctuation of the height $h$ for a lattice width $L$ is expressed by

$$
\sigma(L, h) \sim L^{\alpha} f\left(h / L^{z}\right)
$$

where $\beta$ and $z(=\alpha / \beta)$ are growth and dynamic exponents, respectively, and $\alpha$ is roughness exponent. The function $f(x)$ is a scaling function given by

$$
f(x) \sim \begin{cases}x^{\beta} & \text { for } x \ll 1, \\ \text { const. } & \text { for } x \gg 1\end{cases}
$$

Therefore,

$$
\sigma(L, h) \sim \begin{cases}h^{\beta} & \text { for } h \ll L, \\ L^{\alpha} & \text { for } h \gg L .\end{cases}
$$

These equations are useful to analyze experimental rough interfaces obtained e.g. by ink invasion phenomena [9-11] and burning problems [12-14].

Recently, Cao and Wong [15] discussed heterogeneous Eden growth in order to investigate nonuniversality which is found in experiments of sedimentary sandstones [16,17], lignite coals [18], colloidal silicate particles [19,20] and so on [2]. They measured several dimensions of perimeter, hull and mass of cluster. The differences between the roughness exponents of the original Eden model and their models are subtle and they did not get any clear conclusion especially when the concentration of obstacles is smaller than a critical value. One of the purposes of this paper is to shed light on this problem by introducing well defined quantities like the growth rate and by carrying out finite size analysis.

Secondly, there are various impurities which exert influences of not only reduction, but also acceleration of growth rate. If we consider a forest-fire problem, there are withered and dry trees as well as raw and wet trees. The former accelerates the spreading of fire, while the latter decreases the spreading speed. Obstacles and related pinning phenomena has been studied widely [21,22]. In this paper, however, we discuss only an effect of acceleration in the Eden model. Randomly quenched acceleration sites with concentration $p$ get incorporated into the growing cluster immediately if any of their neighbouring sites get occupied. Obviously, if there is an infinite path through acceleration sites through the sample, the average height of the cluster goes immediately to infinity, i.e. the growth rate becomes infinity.

We have carried out numerical simulations to calculate the growth rate $v$ for various concentrations of acceleration sites. The growth rate increases with the concentration, and diverges at a critical value, which is determined as $p_{c}=0.592 \pm 0.005$ in agreement with the known value for the site percolation threshold of square lattice [23,24]. The growth rate $v$ diverges near to $p_{c}$ according to a power law with an exponent which 
was measured to be $1.33 \pm 0.08$. This value is close to the exponent of the correlation length $v=\frac{4}{3}[23,24]$.

As for the scaling of the surface roughness, we calculate the roughness exponent $\alpha$ which characterizes a self-affine surface. The Eden model has $\alpha=\frac{1}{2}$ and $\beta=\frac{1}{3}$ for the roughness and the growth exponents, respectively. Due to general symmetry considerations one expects that the present Eden model with acceleration sites is found to belong to the same universality class as the usual Eden growth, namely to the KPZ universality class with $\alpha=0.5$ within a range of $p<p_{c}$ [25]. The measured effective exponent varies with $p$, however, a finite size analysis shows that this is a crossover effect and the KPZ universality seems to be valid. At $p=p_{c}$, the surface is not selfaffine, but self-similar, because it is nothing but an ordinary percolation surface. It is, however, difficult to get a definite value of $\beta$ at $p=p_{c}$ in the present model.

In the next section, we discuss the universality of the model. In Section 3 we present a scaling analysis and the paper terminates with a discussion of the results and a conclusion.

\section{Universality of the Eden growth model with acceleration sites}

The model is defined here on the square lattice as an extension of the usual Eden growth process. Before the process starts acceleration sites are positioned randomly on the lattice with probability $p$. The Eden growth proceeds as usual: If a site is neighbor to an occupied site, it becomes a growth site and at every time step one of the growth sites is chosen at random and occupied (added to the cluster). However, if an acceleration site becomes neighbor to an occupied one, it gets immediately part of the cluster.

We consider Eden growth on a square lattice of $L_{x} \times L_{y}\left(L_{x} \ll L_{y}\right)$. One of the horizontal edges is a starting line for Eden growth. Acceleration sites which are distributed randomly in the lattice are denoted by circles in Fig. 1. The hatched region in Fig. 1 show the Eden cell which is already grown. If an Eden cell grows upward at a

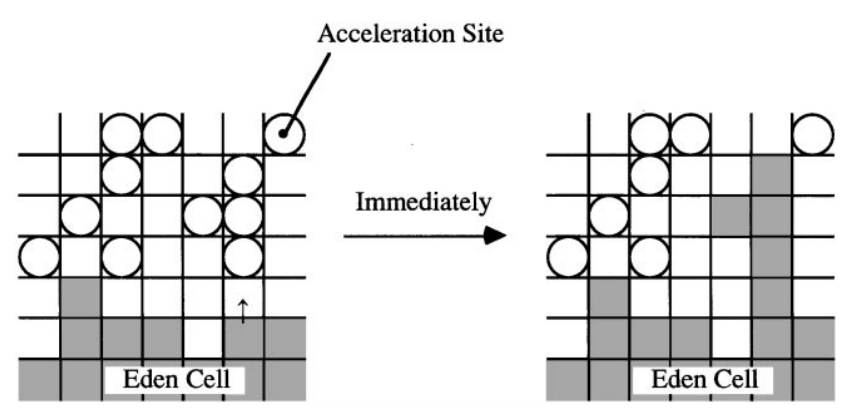

Fig. 1. Eden model with acceleration sites. Hatched region is the grown Eden cells and the site with circle is the acceleration site. If we assume that a cell indicated by an arrow grows, 4 acceleration sites turn to Eden cells immediately. 


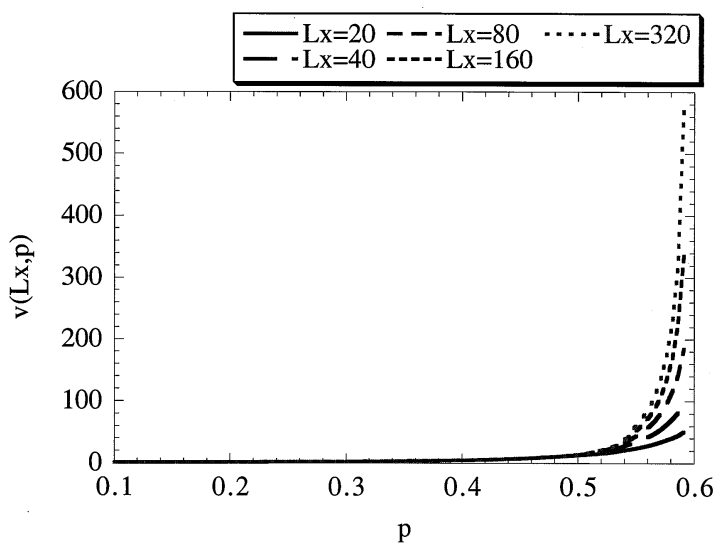

Fig. 2. The growth rate against the concentration of acceleration sites. $L_{x}$ is the horizontal lattice width.

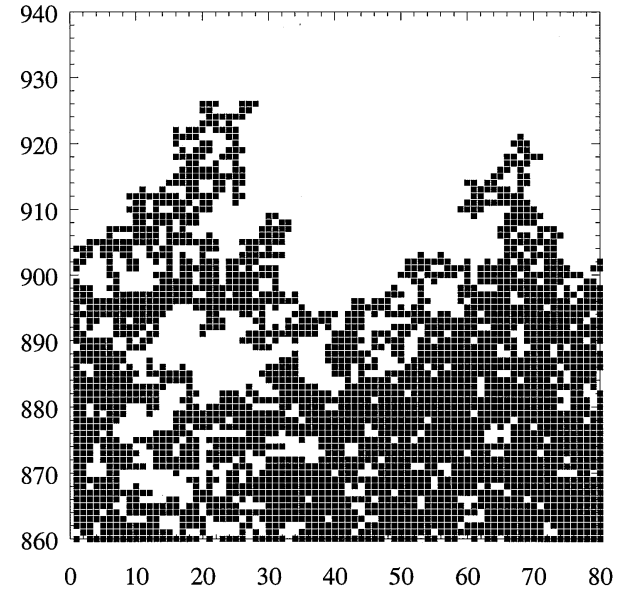

(a)

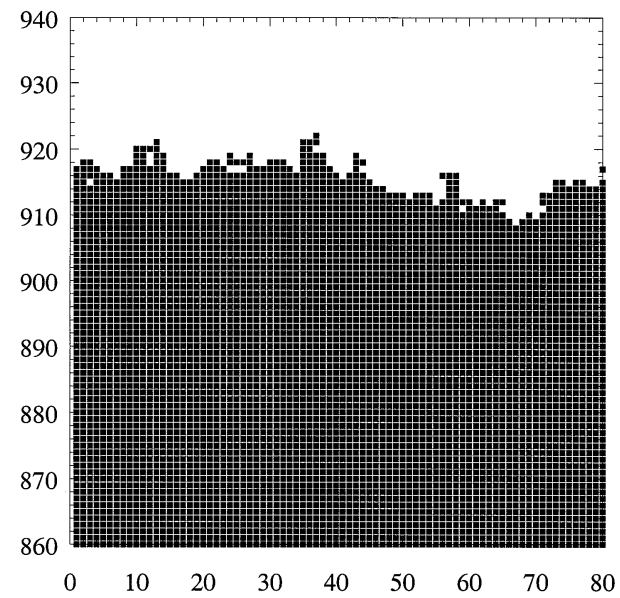

(b)

Fig. 3. Typical patterns of Eden growth surfaces with acceleration sites. The lattice size $L_{x} \times L_{y}$ is $80 \times 1000$. (a) The concentration of acceleration sites is $p=0.5$. (b) $p=0$ (original Eden growth).

site shown by an arrow of Fig. 1 and touches an acceleration site, then the connected acceleration sites with it will be occupied by the Eden cells immediately.

When the number of Eden cells increases by the same number as $L_{x}$, we proceed a unit time. The growth rate of Eden cells is measured by height increase for a unit time for various lattice sizes from $L_{x}=16$ to $L_{x}=320$, where $L_{y}$ is set large enough so as $L_{x} \ll L_{y}$, and shown in Fig. 2 against the concentration of acceleration sites. The growth rate increases with the concentration of acceleration sites and diverges near 0.6. Fig. 3a shows a surface region of $L_{x}=80$ with the concentration of acceleration sites of $p=0.5$, and Fig. $3 \mathrm{~b}$ shows a surface of a regular Eden model for comparison. 


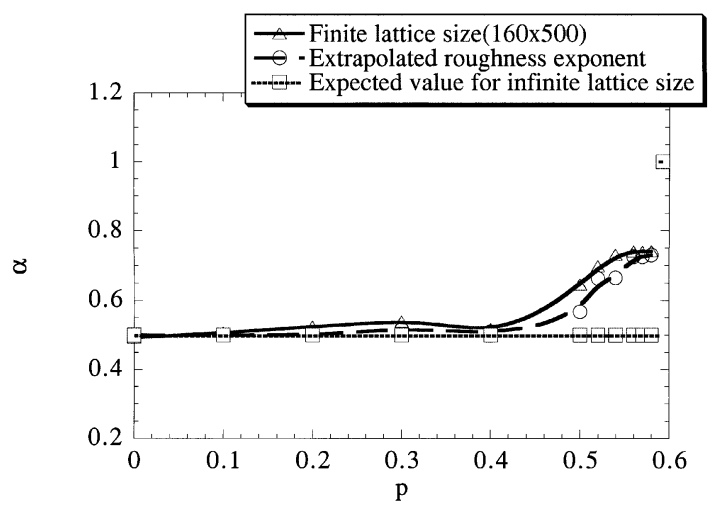

Fig. 4. Roughness exponent $\alpha$ against the concentration of acceleration sites. The triangle shows the results obtained from the data of $L_{x}=160$ and the circle shows the roughness exponents $\alpha$ extrapolated from our results to infinite lattice. The square means the roughness exponents which are expected for the infinite lattice size.

Next, we measure the roughness exponent $\alpha$ which is obtained by the saturated value of fluctuation of the surface height. The raw values of the effective exponent $\alpha$ for $p<p_{c}$ obtained from the data for $L_{x} \leqslant 160$ are shown by the triangle in Fig. 4. Those values become larger gradually with the concentration of acceleration sites. This seems to contradict our expectation about universality of the scaling of the roughness. However, if we calculate the roughness exponents for various lattice sizes of $L_{x}$ from 16 to 320 , as denoted by the circle in Fig. 4 we get a strong indication that the change in the exponent is due to a crossover. For $0.1<p \leqslant 0.5$, the extrapolated value is about 0.5 , and we get $\alpha \approx 0.5$ for $0<p \leqslant 0.1$ already without the extrapolation. For $0.5<p<0.6$, the pattern seems to be self-similar for the considered lattice sizes, but the roughness exponent still decreases, although very slowly, with the increasing lattice width $L_{x}$. From Fig. 4, it seems to be likely that the roughness exponent converges to 0.5 up to the threshold value of concentration of acceleration sites at the infinite lattice size. This means that, although there exist a lot of acceleration sites in the lattice and the pattern differs apparently very much from the original one-dimensional Eden growth model, as long as $p<p_{c}$ the universality class is the same as for the usual Eden growth, namely the KPZ universality class [25].

\section{Scaling treatment}

Let us consider the relation between the growth rate and the concentration of acceleration sites for various lattice sizes shown in Fig. 2. We expect usual finite size scaling similar to Eqs. (1)-(3). The growth rate $v\left(L_{x}, p\right)$ for the lattice width $L_{x}$ and the concentration of acceleration sites $p$ can be expressed as

$$
v\left(L_{x}, p\right) \sim|\varepsilon|^{-s} g\left(|\varepsilon| / L_{x}^{-1 / v}\right) \text { for } p<p_{c},
$$




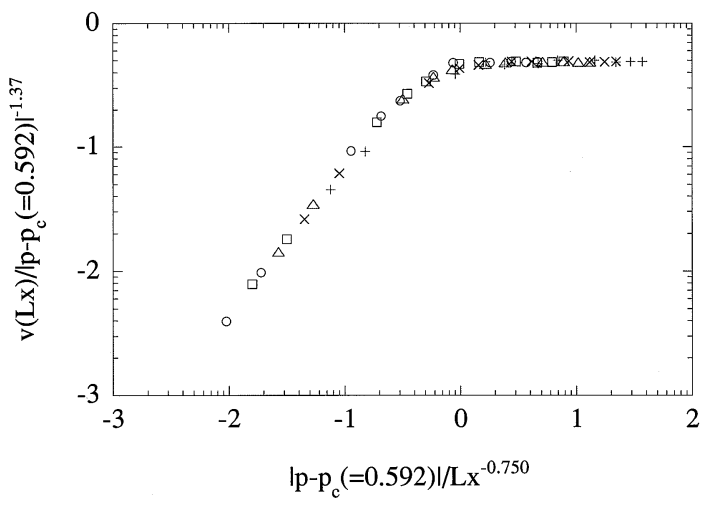

Fig. 5. The data collapse of scaled fluctuation of growth rate is plotted against $\left|p-p_{c}\right| / L_{x}^{-0.750}$ for several values of lattice width $L_{x}$, where the critical concentration of acceleration sites $p_{c}$ is 0.592 .

where $\varepsilon=p-p_{c}$ and the function $g(x)$ is a scaling function given by

$$
g(x) \rightarrow \begin{cases}x^{s} & \text { if } x \rightarrow 0, \\ \text { const. } & \text { if } x \rightarrow \infty,\end{cases}
$$

and $s$ and $v$ are determined by data collapse. Therefore we obtain explicitly

$$
v\left(L_{x}, p\right) \sim \begin{cases}L_{x}^{s / v} & \text { for } \varepsilon \rightarrow 0, \\ |\varepsilon|^{-s} & \text { for } L_{x} \rightarrow \infty\end{cases}
$$

In order to check this relation, we change the scales in Fig. 2 following Eq. (4). Looking for the best collapse of data, we obtain

$$
s=1.37 \pm 0.07, \quad v=1.33 \pm 0.08,
$$

which is shown in Fig. 5. These values of $s$ and $v$ are very close to $\frac{4}{3}$ which is the value of the exponent of the correlation length in ordinary percolation $[23,24]$.

We have also plotted the fluctuation $W$ (standard deviation) of the growth rate against the concentration of acceleration sites for several values of the lattice width of $L_{x}$ in Fig. 6 and the data collapse is checked by assuming the following function,

$$
W(L) \sim|\varepsilon|^{-s^{\prime}} h\left(|\varepsilon| / L_{x}^{-1 / v^{\prime}}\right),
$$

where $h(x)$ is seemed to be expressed by the scaling function

$$
h(x) \rightarrow \begin{cases}x^{-1} & \text { if } x \rightarrow 0, \\ x^{s^{\prime}} & \text { if } x \rightarrow \infty,\end{cases}
$$

and $s^{\prime}$ and $v^{\prime}$ are the exponents determined by the data collapse. The result is shown in Fig. 7 when $s^{\prime}=1.33 \pm 0.11$ and $v^{\prime}=1.33 \pm 0.10$ as the best data collapse. 


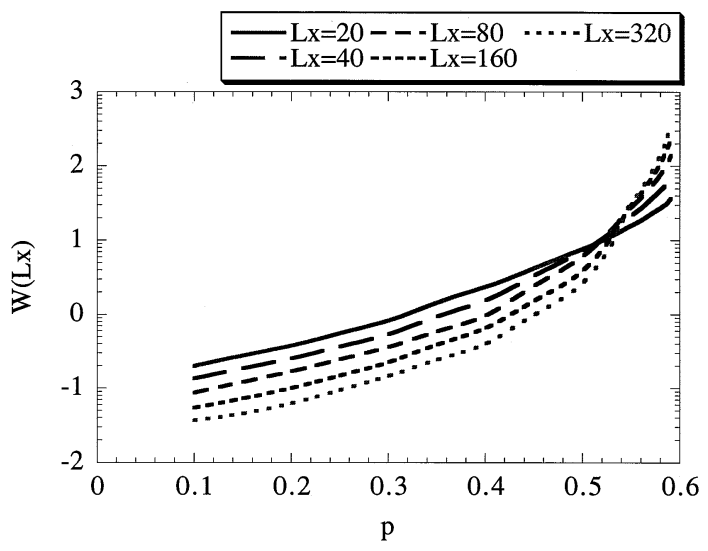

Fig. 6. The fluctuation of growth rate is plotted against the concentration of acceleration sites for several values of lattice width $L_{x}$.

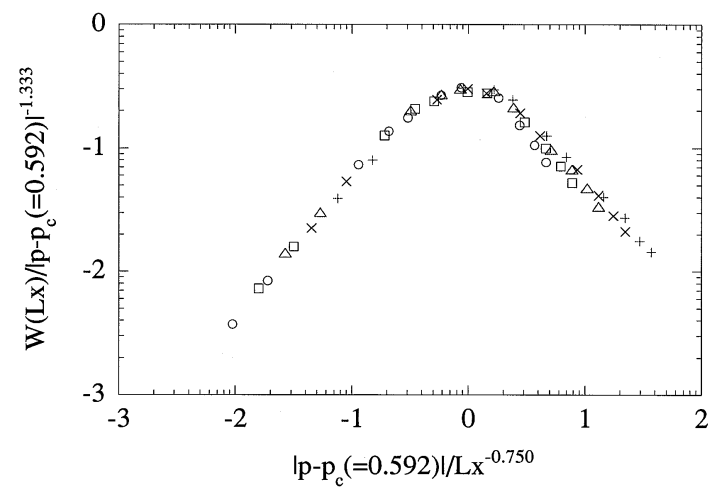

Fig. 7. The data collapse of scaled fluctuation of growth rate is plotted against $\left|p-p_{c}\right| / L_{x}^{-0.750}$ for several values of lattice width $L_{x}$, where the critical concentration of acceleration sites $p_{c}$ is 0.592 .

\section{Conclusions}

We have discussed a new Eden growth model which has randomly distributed acceleration sites which immediately become a part of the Eden cluster whenever they become neighbor to an occupied site. Therefore, the Eden growth is accelerated by the acceleration site, and as the concentration of such sites increases, the growth rate speeds up and becomes infinite at the percolation threshold. This divergence of growth rate can be described by the same exponent as for the correlation length exponent of the percolation model.

We have also been interested in the problem of universality of the surface scaling of the present modified Eden model. As seen in Fig. 4, we have obtained $\alpha=0.50 \pm 0.01$ for $p<0.5$, which indicates that the present modified Eden model belongs to the same 
universality class as the original Eden model. It is difficult to get a definite exponent for $0.5<p<p_{c}=0.5927 \ldots$ from sizes studied here, because in this range the roughness exponent decreases very slowly with the lattice size. However, there are no physical factors which would cause any transition between $0.5<p<p_{c}$ and, therefore, we expect that in the thermodynamic limit even in this range the exponent approaches its universal value. The present modified Eden surface is self-affine for $p<p_{c}$ as the original Eden surface, but at $p=p_{c}$ the present model turns to have a self-similar surface. It becomes the infinite percolation cluster at the threshold.

In order to check our prediction experimentally, we have already prepared a laboratory experiment which corresponds to the Eden model with acceleration sites. The results of this experiment will be published in a separate paper.

\section{Acknowledgements}

The authors thank Prof. S. Havlin for reading and correcting the manuscript, and S.M. and J.K. thank for the warm hospitality at BU. This work was partly supported by the Scientific Research Fund of Ministry of Education, Science and Culture of Japan, the Foundation for Private Universities in Japan and OTKA (T016568) and US-Hungarian Joint Fund (MAKA93b-352).

\section{References}

[1] T. Vicsek, Fractal Growth Phenomena, 2nd ed., World Scientific, Singapore, 1989.

[2] A.-L. Barabási, H.E. Stanley, Fractal Concepts in Surface Growth, Cambridge University Press, Cambridge, 1995.

[3] P. Meakin, P. Ramanlal, L.M. Sander, R.C. Ball, Phys. Rev. A 34 (1986) 5091.

[4] M. Eden, in: F. Family, T. Vicsek (Eds.), Dynamics of Fractal Surfaces, World Scientific, Singapore, 1991.

[5] J. Kertész, D.E. Wolf, J. Phys. A 21 (1988) 747.

[6] S. Miyazima, A. Bunde, S. Havlin, H.E. Stanley, J. Phys. A 19 (1986) L1159.

[7] A. Bunde, S. Miyazima, H.E. Stanley, J. Stat. Phys. 47 (1987) 1.

[8] F. Family, T. Vicsek, J. Phys. A 18 (1985) L75.

[9] S.V. Buldyrev, A.-L. Barabási, F. Caserta, S. Havlin, H.E. Stanley, T. Vicsek, Phys. Rev. A 45 (1992) R8313.

[10] S.V. Buldyrev, S. Havlin, J. Kertész, R. Sadr-Lahijany, A. Shehter, H.E. Stanley, Phys. Rev. E 52 (1995) 373.

[11] T. Nagamine, K. Maruyama, S. Miyazima, Fractals, vol. 1, World Scientific, Singapore, 1993, p. 998.

[12] J. Zhang, Y.-C. Zhang, P. Alstrøm, M.T. Levinsen, in: R. Jullien, J. Kertész, P. Meakin, D.E. Wolf (Eds.), Surface Disordering: Growth, Roughning and Phase Transition, Nova Science, New York, 1992.

[13] J. Zhang, Y.-C. Zhang, P. Alstrøm, M.T. Levinsen, Physica A 189 (1992) 383.

[14] Y. Ueno, T. Nagamine, K. Maruyama, S. Miyazima, Physica A 233 (1996) 672.

[15] Qi-zhong Cao, Po-zen Wong, Phys. Rev. Lett. 67 (1991) 77.

[16] Po-zen Wong, Phys. Today 41 (1988) 24.

[17] A.H. Thompson, A.J. Katz, C.E. Krohn, Adv. Phys. 36 (1987) 625.

[18] P.W. Schmidt, in: D. Avnir (Ed.), Fractal Approach to Heterogeneous Chemistry, Wiley, New York, 1989, p. 67.

[19] D. Keefer, D.W. Schaefer, Phys. Rev. Lett. 56 (1986) 2376. 
[20] K.D. Keefer, in: C.J. Brinker, D.E. Clark, D.R. Ulrich (Eds.), Better Ceramics Through Chemistry II, MRS Symp. Proc., vol. 73, Materials Research Society, Pittsburgh, 1986, p. 295.

[21] S.V. Buldyrev, A.-L. Barabási, S. Havlin, J. Kertész, H.E. Stanley, H.S. Xenias, Physica A 191 (1992) 220.

[22] L.H. Tang, H. Leschhorn, Phys. Rev. A 45 (1992) R8309.

[23] D. Stauffer, A. Aharony, Introduction to Percolation Theory, Taylor and Francis, London, 1992.

[24] A. Bunde, S. Havlin, Fractals and Disordered System, 2nd ed., Springer, Heidelberg, 1996.

[25] M. Kardar, G. Parisi, Y.-C. Zhang, Phys. Rev. Lett. 56 (1986) 889. 